Moroccan J. of Pure and Appl. Anal. (MJPAA)

Volume 8(1), 2022, Pages 28-43

ISSN: Online 2351-8227 - Print 2605-6364

DOI: $10.2478 / \mathrm{mjpaa}-2022-0003$

\title{
The ideal of Lipschitz classical $p$-compact operators and its injective hull
}

\author{
TOUfiK Tiaiba $^{1}$, Dahmane Achour ${ }^{2}$
}

Авstract. We introduce and investigate the injective hull of the strongly Lipschitz classical $p$-compact operator ideal defined between a pointed metric space and a Banach space. As an application we extend some characterizations of the injective hull of the strongly Lipschitz classical $p$-compact from the linear case to the Lipschitz case. Also, we introduce the ideal of Lipschitz unconditionally quasi $p$-nuclear operators between pointed metric spaces and show that it coincides with the Lipschitz injective hull of the ideal of Lipschitz classical $p$-compact operators.

Mathematics Subject Classification (2020). 47L20, 47H99, 47B10, 26A16, 46A45.

Key words and phrases. Classical $p$-compact operator, Lipschitz classical $p$-compact operator, Lipschitz operator ideal, Lipschitz injective hull, Lipschitz unconditionally quasi $p$-nuclear operator.

\section{Introduction}

Since the article of Farmer and Johnson [8], which deals with a class of Lipschitz operators, the $p$-summing Lipschitz operators, several authors have studied different classes of Lipschitz operators which, in some sense, extends linear Banach operator ideals (see e.g. [2, 5, 13]). Moreover, the concept of Lipschitz Banach operator ideals defined between a pointed metric space and a Banach space was introduced in [3], extending the Banach linear operator ideals

Received November 6, 2020 - Accepted April 30, 2021.

(C) The Author(s) 2021. This article is published with open access by Sidi Mohamed Ben Abdallah University.

${ }^{1}$ Laboratory of Functional Analysis and Geometry of Spaces, University of Msila, PB 166 Msila 28000, Algeria.

e-mail: toufik.tiaiba@univ-msila.dz

${ }^{2}$ Laboratory of Functional Analysis and Geometry of Spaces, University of Msila, PB 166 Msila 28000, Algeria. e-mail: dahmane.achour@univ-msila.dz (Corresponding Author). 
and there is also the injective hull procedure of Banach Lipschitz operator ideals [3, Definition 2.2] which were introduced to generate "new" Lipschitz Banach operator ideals from previous one, which extends the "procedure" in the case of linear operators. On the other hand, given a Lipschitz operator ideal defined between pointed metric spaces, Achour et al. [1] defined a procedure which assign new Lipschitz operator ideals from a given one, named the Lipschitz injective hull of Lipschitz operator ideals. In [1, Proposition 2.7] the authors prove that both procedure (Lipschitz injective hull procedure and the injective hull procedure of Banach Lipschitz operator ideals) are in general different.

The theory of $p$-compact operators was initiated by Pietsch [16, 18.3.1 and 18.3.2] and Fourie and Swart [11]. There is another well-known notion of $p$-compact operators, which is a recent notion, introduced and studied in 2002 by Sinha and Karn [17]. This last was extended to the Lipschitz case by Achour et al. [2]. Following [15], we call classical $p$-compact operators to the $p$-compact operators of Fourie and Swart . The family of classical $p$-compact operators is a Banach operator ideal [11] and will be denoted by $\left(\mathcal{C K}_{p},\|\cdot\|_{\mathcal{C K}_{p}}\right)$. Among many important properties, it is shown in [10] (see also, for the more general case [9]) that the injective hull of the classical $p$-compact operators coincides with the unconditionally quasi $p$-nuclear operators of Kim. Unconditionally $p$-compact was introduced and studied by Kim [14]. In the same paper [14] the author also proves that an operator is unconditionally quasi $p$-nuclear, if and only if its adjoint is unconditionally $p$-compact.

In the present paper, we deal with a new class of Lipschitz operator ideals which extend the classical $p$-compact operators of Fourie and Swart and its injective hull [11, 10]. In [5] the notion of Lipschitz ideal of (strongly) Lipschitz $(p, r, s)$-nuclear operator was introduced and in this article the ideal of (strongly) Lipschitz $\left(\infty, p, p^{*}\right)$-nuclear operators will be call (strongly) Lipschitz classical $p$-compact.

The article is organized as follows. In Section 2, we introduce and study the injective hull of the Banach ideal of strongly Lipschitz classical $p$-compact operator ideal defined between a pointed metric space and a Banach space (also, called strongly Lipschitz $\left(\infty, p, p^{*}\right)$-nuclear operator ideal, defined in [5]). We prove that the injective hull of strongly Lipschitz classical $p$-compact coincides with the Lipschitz composition ideal with the injective hull of classical $p$ compact linear operators. As an application, we extend some characterizations of the injective hull of the Lipschitz operator ideal $\mathcal{C K}_{s t, p}^{L}$ (which can be seen as a generalization of the linear case). For the case $p=\infty$ we give a Lipschitz version of the well-known theorem of to Terzioğlu [18, Theorem 1]. In Section 3, the notion of factorable Lipschitz unconditionally quasi $p$-nuclear operators between a pointed metric space and a Banach space is introduced, showing that a Lipschitz map is factorable unconditionally quasi $p$-nuclear, if and only if its adjoint is unconditionally $p$-compact. Finally, in the last section, we introduce the ideal of Lipschitz unconditionally quasi $p$-nuclear operators between pointed metric spaces and show that it coincides with the Lipschitz injective hull of the ideal of Lipschitz classical p-compact operators.

Our notation is standard. $X$ and $Y$ will be pointed metric spaces with a base point denoted by 0 and the metric will be denoted by $d$. We denote by $B_{X}$ the closure of the ball centered at 0 with radius 1 . Also, $E$ and $F$ will stand for Banach spaces over the same field $\mathbb{K}$ (either $\mathbb{R}$ or $\mathrm{C}$ ). A Banach space $E$ will be considered as pointed metric space with a base point 0 and 
distance $d\left(x, x^{\prime}\right)=\left\|x-x^{\prime}\right\|$. With $\operatorname{Lip}_{0}(X, Y)$ we denote the set of all Lipschitz mappings from $X$ to $Y$ such that maps 0 to 0 and we put

$$
\operatorname{Lip}(T)=\inf \left\{C>0: d\left(T x, T x^{\prime}\right) \leq C d\left(x, x^{\prime}\right) ; \forall x, x^{\prime} \in X\right\} .
$$

In particular, $\operatorname{Lip}_{0}(X, E)$ is the Banach space of all Lipschitz mappings $T$ from $X$ to $E$ that vanish at 0 , under the Lipschitz norm $\operatorname{Lip}(\cdot)$. When $E=\mathbb{K}, \operatorname{Lip}_{0}(X, \mathbb{K})$ is denoted by $X^{\#}$ and it is called the Lipschitz dual of $X$. The space of all bounded linear operators from $E$ to $F$ is denoted by $\mathcal{L}(E, F)$ and it is a Banach space with the usual supremum norm. The continuous dual space $\mathcal{L}(E, \mathbb{K})$ of $E$ is denoted by $E^{*}$, and $\mathcal{K}(E, F)$ is the space of compact linear operators. It is clear that $\mathcal{L}(E, F)$ is a subspace of $\operatorname{Lip}_{0}(E, F)$ and, in particular, $E^{*}$ is a subspace of $E^{\#}$.

Let $X$ be a metric space. A molecule on $X$ is a scalar valued function $m$ on $X$ with finite support that satisfies $\sum_{x \in X} m(x)=0$. We denote by $\mathcal{M}(X)$ the linear space of all molecules on $X$. For $x, x^{\prime} \in X$ the molecule $m_{x x^{\prime}}$ is defined by $m_{x x^{\prime}}=\chi_{\{x\}}-\chi_{\left\{x^{\prime}\right\}}$, where $\chi_{A}$ is the characteristic function of the set $A$. For $m \in \mathcal{M}(X)$ we can write $m=\sum_{j=1}^{n} \lambda_{j} m_{x_{j} x_{j}^{\prime}}$ for some suitable scalars $\lambda_{j}$, and we write

$$
\|m\|_{\mathcal{M}(X)}=\inf \left\{\sum_{j=1}^{n}\left|\lambda_{j}\right| d\left(x_{j}, x_{j}^{\prime}\right), m=\sum_{j=1}^{n} \lambda_{j} m_{x_{j} x_{j}^{\prime}}\right\},
$$

where the infimum is taken over all representations of the molecule $m$. Denote by $Æ(X)$ the completion of the normed space $\left(\mathcal{M}(X),\|\cdot\|_{\mathcal{M}(X)}\right)$. This space was first introduced by Arens and Eells [4] in 1956. The terminology Arens-Eells space $Æ(X)$ is due to Weaver [19]. The ArensEells space is also known as the Lipschitz-free Banach space of a metric space X. For more of this space, we refer the reader to the manuscript [12] and the reference therein. If we consider the canonical Lipschitz isometry $\delta_{X}: X \longrightarrow Æ(X)$ given by $\delta_{X}(x):=m_{x 0}, x \in X$, then for an operator $T \in \operatorname{Lip}_{0}(X, E)$, there exists a unique linear map $T_{L}: \nexists(X) \longrightarrow E$ such that $T=T_{L} \delta_{X}$ and $\left\|T_{L}\right\|=\operatorname{Lip}(T)$. The operator $T_{L}$ is referred to as the linearization of $T$ (see for instance [19, Theorem 2.2.4 (b)]). The correspondence $T \longleftrightarrow T_{L}$ establishes an isometric isomorphism between the Banach spaces $\operatorname{Lip}_{0}(X, E)$ and $\mathcal{L}(\nexists(X), E)$. In particular, the spaces $X^{\#}$ and $Æ(X)^{*}$ are isometrically isomorphic via the linearization $R(f):=f_{L}$, where $f_{L}(m)=\sum_{x \in X} f(x) m(x)$.

Following [3], an ideal of Lipschitz mappings $\mathcal{I}_{\text {Lip }}$ is a subclass of the class of all Lipschitz mappings between pointed metric space and Banach space such that for a pointed metric space $X$ and Banach space $E$, the components

$$
\mathcal{I}_{\text {Lip }}(X, E):=\operatorname{Lip}_{0}(X, E) \cap \mathcal{I}_{\text {Lip }}
$$

is a vector subspace of $\operatorname{Lip}_{0}(X, E)$ that is invariant by the composition of a linear operator on the right and Lipschitz operator on the left and which contains the Lipschitz finite rank mappings type.

Let $1 \leq p \leq \infty$, we write $p^{*}$ the conjugate index of $p$, that is $1 / p+1 / p^{*}=1$. As usual $\ell_{p}(E)$ denotes the vector space of all absolutely $p$-summable sequences, with the usual norm $\|\cdot\|_{p}$ and $\ell_{p}^{\omega}(E)$ the space of all weakly $p$-summable sequences with the norm $\left\|\left(x_{n}\right)_{n}\right\|_{p}^{\omega}=$ 
$\sup _{x^{*} \in B_{X^{*}}}\left\|\left(\left\langle x_{n}, x^{*}\right\rangle\right)_{n}\right\|_{p}$. The closure in $\ell_{p}^{\omega}(E)$ of the set of all sequences in $E$ which have only a finite number of non-zero terms, is a Banach space with respect to the norm $\|\cdot\|_{p}^{\omega}$. We denote this space by $\ell_{p}^{u}(E)$.

Let $\left(f_{j}\right)_{j}$ be a sequence in $X^{\#}$. Following [5], the sequence $\left(f_{j}\right)_{j}$ is Lipschitz $\omega^{*}$-p-summable if there is a constant $C$ such that for all $n \in \mathbb{N}$ and for all $x, x^{\prime} \in X$ we have

$$
\left\|\left(f_{j}(x)-f_{j}\left(x^{\prime}\right)\right)_{j=1}^{n}\right\|_{p} \leq C d\left(x, x^{\prime}\right) .
$$

The smallest such constant $C$ will be denoted by $\left\|\left(f_{j}\right)_{j}\right\|_{p}^{L, \omega^{*}}$ and $\ell_{p}^{L, \omega^{*}}\left(X^{\#}\right)$ will denote the set of Lipschitz $\omega^{*}$-p-summable sequences in $X^{\#}$. Clearly

$$
\left\|\left(f_{j}\right)_{j}\right\|_{p}^{L, \omega^{*}}=\sup _{\substack{x, x^{\prime} \in X \\ x \neq x^{\prime}}} \frac{\left\|\left(f_{j}(x)-f_{j}\left(x^{\prime}\right)\right)_{j}\right\|_{p}}{d\left(x, x^{\prime}\right)} .
$$

A sequence $\left(f_{j}\right)$ in $X^{\#}$ is called Lipschitz $\omega^{*}$-unconditionally $p$-summable if

$$
\sup _{\substack{x, x^{\prime} \in X \\ x \neq x^{\prime}}} \frac{\left(\sum_{j=n}^{+\infty}\left|f_{j}(x)-f_{j}\left(x^{\prime}\right)\right|^{p}\right)^{\frac{1}{p}}}{d\left(x, x^{\prime}\right)} \underset{n}{\longrightarrow} 0 .
$$

The set of all Lipschitz $\omega^{*}$-unconditionally $p$-summable sequences in $X^{\#}$ is denoted by $\ell_{p}^{L, \omega^{*}, u}\left(X^{\#}\right)$.

It is a well known result that the canonical correspondence $T \mapsto\left(\left\langle e_{j}^{*}, T(\cdot)\right\rangle\right)_{j}$, provides an isometric isomorphism of $\operatorname{Lip}_{0}\left(X, \ell_{p}\right)$ onto $\ell_{p}^{L, \omega^{*}}\left(X^{\#}\right)$. Also, $\left[\ell_{p}^{L, \omega^{*}}\left(X^{\#}\right),\|\cdot\|_{p}^{L, \omega^{*}}\right]=\left[\ell_{p}^{\omega}\left(X^{\#}\right),\|\cdot\|_{p}^{\omega}\right]$ (see [5, Lemma 2.4]).

Following [13] a Lipschitz map $T \in \operatorname{Lip}_{0}(X, E)$ is Lipschitz compact if the set

$$
\left\{\frac{T x-T x^{\prime}}{d\left(x, x^{\prime}\right)}, x, x^{\prime} \in X, x \neq x^{\prime}\right\}
$$

is relatively compact in $E$. Denote by $\operatorname{Lip}_{0 \mathcal{K}}(X, E)$ the set of Lipschitz compact operators from $X$ to $E$. Belacel and Chen [5, Lemma 3.5] proved that the canonical correspondence $T \mapsto\left(\left\langle e_{j}^{*}, T(\cdot)\right\rangle\right)_{j}$, provides an isometric isomorphism of $\operatorname{Li} p_{0 \mathcal{K}}\left(X, \ell_{p}\right)$ onto $\ell_{p}^{L, \omega^{*}, u}\left(X^{\#}\right)$ when $1 \leq p<\infty$. For $p=\infty$, the isometric isomorphism is from $\operatorname{Lip}_{0 \mathcal{K}}\left(X, c_{0}\right)$ onto $\ell_{\infty}^{L, \omega^{*}, u}\left(X^{\#}\right)$. Furthermore, $\left[\ell_{p}^{L, \omega^{*}, u}\left(X^{\#}\right),\|\cdot\|_{p}^{L, \omega^{*}}\right]=\left[\ell_{p}^{u}\left(X^{\#}\right),\|\cdot\|_{p}^{\omega}\right]$ and $\ell_{\infty}^{L, \omega^{*}, u}\left(X^{\#}\right)=c_{0}\left(X^{\#}\right)=\ell_{\infty}^{u}\left(X^{\#}\right)$ for $p=\infty$.

Let $1 \leq p \leq \infty$ we say that an operator $T: E \longrightarrow F$ is called classical $p$-compact (see [16, 18.3.1 and 18.3.2] or [11]) if there are compact operators $P: E \longrightarrow \ell_{p}$ and $Q: \ell_{p} \longrightarrow F$ such that $T=Q \circ P$. The norm on the vector space $\mathcal{C} \mathcal{K}_{p}(E, F)$ of all classical $p$-compact operators from $E$ to $F$ is defined by $\|T\|_{\mathcal{C K}_{p}}=\inf \left\{\|P\|\|Q\|, P \in \mathcal{K}\left(E, \ell_{p}\right), Q \in \mathcal{K}\left(\ell_{p}, F\right)\right.$ and $\left.T=Q \circ P\right\}$. It is shown in [11] that $\left(\mathcal{C K}_{p},\|\cdot\|_{C \mathcal{K}_{p}}\right)$ is a Banach operator ideal and that $T \in \mathcal{C} \mathcal{K}_{p}(E, F)$ if and only if $T$ has a representation $T=\sum_{n=1}^{+\infty} x_{n}^{*}(\cdot) y_{n}$, where $\left(x_{n}^{*}\right)_{n} \in \ell_{p}^{u}\left(E^{*}\right)$ and $\left(y_{n}\right)_{n} \in \ell_{p^{*}}^{u}(F)$. 
Also, $\|T\|_{\mathcal{C K}_{p}}=\inf \left\{\left\|\left(x_{n}^{*}\right)_{n}\right\|_{p}^{\omega}\left\|\left(y_{n}\right)_{n}\right\|_{p^{*}}^{\omega}\right\}$, with the infimum taken over all representations of $T$ as above. The notion of unconditionally quasi $p$-nuclear operators was introduced by Kim [14, p. 136]. For $1 \leq p<\infty$, a linear map $T$ between Banach spaces $E$ and $F$ is said to be unconditionally $p$-nuclear if there exists a sequence $\left(x_{n}^{*}\right)_{n}$ in $\ell_{p}^{u}\left(E^{*}\right)$ such that $\|T x\| \leq$ $\left\|\left(x_{n}^{*}(x)\right)_{n}\right\|_{p}$ for all $x \in E$. This class, which is denoted by $\mathcal{N}_{u p}^{Q}$, endowed with the norm $v_{u p}^{Q}$ defined as the infimum of $\left\|\left(x_{n}^{*}\right)_{n}\right\|_{p}^{\omega}$ taken over all the sequences $\left(x_{n}^{*}\right)_{n}$ satisfying the above inequality, becomes a Banach operator ideal.

\section{The ideal of strongly Lipschitz classical $p$-compact operators and its injective hull}

In [5] the concept of strongly Lipschitz classical $p$-compact is introduced and studied, these operators were called $p$-compact. Refer to [5, Definition 3.1 and Theorem 3.2] for the following definition.

Definition 2.1. Let $1 \leq p \leq \infty$. An operator $T \in \operatorname{Lip}_{0}(X, E)$ is called strongly Lipschitz classical p-compact if $T$ has a factorization $T=R S$, where $S \in \operatorname{Lip} p_{0 \mathcal{K}}\left(X, \ell_{p}\right)$ and $R \in \mathcal{K}\left(\ell_{p}, E\right)$ (also, $T$ is called strongly Lipschitz $\left(\infty, p, p^{*}\right)$-nuclear). For a pointed metric space $X$ and a Banach space $E$ the norm on the vector space $\mathcal{C} \mathcal{K}_{s t, p}^{L}(X, E)$ of all strongly Lipschitz classical p-compact operators from $X$ to $E$ is defined by

$$
\|T\|_{\mathcal{C} \mathcal{K}_{s t, p}^{L}}:=\inf \left\{\operatorname{Lip}(S)\|R\|: S \in \operatorname{Lip}_{0 \mathcal{K}}\left(X, \ell_{p}\right), R \in \mathcal{K}\left(\ell_{p}, E\right) \text { and } T=R \circ S\right\} .
$$

It is shown in $\left[5\right.$, Corollary 3.7 (i)] that $T \in \mathcal{C} \mathcal{K}_{s t, p}^{L}(X, E)$ if and only if $T$ has a representation

$$
T x=\sum_{j=1}^{+\infty} f_{j}(x) y_{j}, \forall x \in X
$$

where $\left(f_{j}\right)_{j} \in \ell_{p}^{L, \omega^{*}, u}\left(X^{\#}\right)$ and $\left(y_{j}\right)_{j} \in \ell_{p^{*}}^{u}(E)$. Also,

$$
\|T\|_{\mathcal{C K}_{s t, p}^{L}}=\inf \left\{\left\|\left(f_{j}\right)_{j}\right\|_{p}^{L, \omega^{*}}\left\|\left(y_{j}\right)_{j}\right\|_{p^{*}}^{\omega}\right\}
$$

with the infimum taken over all representations of $T$ as in (2.1). Here it is assumed that $\ell_{\infty}$ is replaced by $c_{0}$ in the case of $p=\infty$. It is shown in [5] that the factorization condition for $T \in \mathcal{C} \mathcal{K}_{s t, p}^{L}(X, E)$ can be relaxed to $T=R \circ S$ where either $S \in \operatorname{Lip} p_{0 \mathcal{K}}\left(X, \ell_{p}\right)$ and $R \in \mathcal{L}\left(\ell_{p}, E\right)$, for $1 \leqslant p \leqslant \infty$ or $S \in \operatorname{Lip}_{0}\left(X, \ell_{p}\right)$ and $R \in \mathcal{K}\left(\ell_{p}, E\right)$, for $1<p \leqslant \infty$. Moreover $\|T\|_{\mathcal{C} \mathcal{K}_{s t, p}^{L}}:=$ $\inf \{\operatorname{Lip}(S)\|R\|\}$, where the infimum is taken over all possible factorizations of $T$ as above.

The next theorem is a particular case of the Proposition 2.12 in [5].

Theorem 2.1. Let $X$ be a pointed metric space, E be a Banach space and let $1 \leq p \leq \infty$. An operator $T \in \operatorname{Lip}_{0}(X, E)$ is strongly Lipschitz classical p-compact if and only if its Linearization $T_{L}: \nexists(X) \longrightarrow$ $E$ is linear classical p-compact. Moreover, we have

$$
\|T\|_{\mathcal{C} \mathcal{K}_{s t, p}^{L}}=\left\|T_{L}\right\|_{\mathcal{C} \mathcal{K}_{p}}
$$


Let $\mathcal{A}$ be a linear Banach operator ideal. A Lipschitz mapping $T \in \operatorname{Lip}_{0}(X, E)$ belongs to the composition Lipschitz operator ideal $\mathcal{A} \circ \operatorname{Lip}_{0}$ if its linearization $T_{L}$ belongs to $\mathcal{A}(Æ(X), E)$ (see $[3$, Definition 3.1]). Moreover, $\mathcal{A} \circ \operatorname{Lip}_{0}$ endowed with the norm $\|T\|_{\mathcal{A} \circ L i p_{0}}=\left\|T_{L}\right\|_{\mathcal{A}}$ is a Banach Lipschitz operator ideal. This way to obtain a Banach Lipschitz operator ideal from a Banach operator ideal is called composition method and the Lipschitz Banach operator ideals obtained in this way are called ideals of composition type.

By Theorem 2.1 and the above criterion, we have the following.

Proposition 2.1. The class $\mathcal{C} \mathcal{K}_{\text {st, }}^{L}$ is the Banach Lipschitz operator ideal generated by the composition method from the Banach operator ideal $\mathcal{C K}_{p}$. In other words

$$
\mathcal{C K}_{s t, p}^{L}(X, E)=\mathcal{C K}_{p} \circ \operatorname{Lip}_{0}(X, E) \text { isometrically }
$$

for every pointed metric space $X$ and every Banach space $E$.

In [3], the authors introduced the injective hull of a Banach Lipschitz operator ideal. For this, recall that for a Banach space $E$, we may consider the linear isometry $\iota_{E}: E \longrightarrow \ell_{\infty}\left(B_{E^{*}}\right)$. Then, following [3, Definition 2.2 (1)], for a Banach Lipschitz operator ideal $\mathcal{I}_{\text {Lip }}$, a pointed metric space $X$ and a Banach space $E$, a Lipschitz operator $T: X \rightarrow E$ belongs to $\mathcal{I}_{\text {Lip }}^{\text {inj }}(X, E)$ if and only if $\iota_{E} T$ belongs to $\mathcal{I}_{\text {Lip }}\left(X, \ell_{\infty}\left(B_{E^{*}}\right)\right)$ with $\|T\|_{\mathcal{I}_{\text {Lip }}}=\left\|\iota_{E} T\right\|_{\mathcal{I}_{\text {Lip }}}$. If $\mathcal{I}_{\text {Lip }}$ is a Banach Lipschitz operator ideal, then $\mathcal{I}_{\text {Lip }}^{\text {inj }}$ is also a Banach Lipschitz operator ideal.

As $\mathcal{C} \mathcal{K}_{s t, p}^{L}$ is a Banach Lipschitz operator ideal of composition type (Proposition 2.1), then from Proposition 2.4 in [1] and Theorem 4.4 in [10], we have the following.

Corollary 2.1. Let $X$ be a pointed metric space and E be a Banach space. Then

$$
\begin{aligned}
\left(\mathcal{C K}_{s t, p}^{L}\right)^{i n j} & =\left(\mathcal{C} \mathcal{K}_{p} \circ \operatorname{Lip}_{0}\right)^{i n j} \\
& =\left(\mathcal{C} \mathcal{K}_{p}\right)^{i n j} \circ \operatorname{Lip}_{0} \\
& =\mathcal{N}_{u p}^{Q} \circ \operatorname{Lip}_{0},
\end{aligned}
$$

where $\mathcal{N}_{u p}^{Q}$ is the Banach ideal of unconditionally p-nuclear operators.

From Corollary 3.5 in [10], we get

Corollary 2.2. Let $X$ be a pointed metric space and $E$ be a Banach space, where $E$ is injective (has the extension property). Then $\mathcal{C} \mathcal{K}_{\text {st, },}^{L}(X, E)=\left(\mathcal{C} \mathcal{K}_{s t, p}^{L}\right)^{i n j}(X, E)$ with $\|T\|_{\mathcal{C}_{s t, p}^{L}}^{L}=\|T\|_{\left(\mathcal{C} \mathcal{K}_{s t, p}^{L}\right)^{i n j}}$.

From Corollary 2.1 and Proposition 3.4 in [10], we get

Proposition 2.2. Let $1 \leq p \leq \infty, X$ be a pointed metric space and $E$ be a Banach space, the following are equivalent.

(1) $T \in\left(\mathcal{C K}_{\text {st, }}^{L}\right)^{i n j}$.

(2) There exists a closed subspace $Z$ of $\ell_{p}$ with $0 \in Z$ such that $T=R \circ S$ for some $S \in \operatorname{Lip} 0 \mathcal{K}(X, Z)$ and $R \in \mathcal{K}(Z, E)$. 
(3) There exists a closed subspace $Z$ of $\ell_{p}$ with $0 \in Z$ such that $T=R \circ S$ for some $S \in \operatorname{Lip} 0 \mathcal{K}(X, Z)$ and $R \in \mathcal{L}(Z, E)$.

Moreover, $\|T\|_{\left(\mathcal{C K}_{s t, p}^{L}\right)^{i n j}}=\inf \{\|R\| \operatorname{Lip}(S): T=R \circ S\}$ where the infimum is taken over all factorizations as above.

From Lemma 3.5 in [5] and Corollary 2.6 (i) in [13] we infer the following identifications.

Lemma 2.1. Let $X$ be a pointed metric space, E be a Banach space and let $1 \leq p \leq \infty$. Then the map $\left(f_{n}\right)_{n} \longmapsto\left(\left(f_{n}\right)_{L}\right)_{n}$ is an isometric isomorphism between the following spaces:

i) From $\left[\ell_{p}^{L, \omega^{*}, u}\left(X^{\#}\right),\|\cdot\|_{p}^{L, \omega^{*}}\right]$ onto $\left[\ell_{p}^{u}\left(Æ(X)^{*}\right),\|\cdot\|_{p}^{\omega}\right]$ for $1 \leq p<\infty$.

ii) From $\ell_{\infty}^{L, \omega^{*}, u}\left(X^{\#}\right)=c_{0}\left(X^{\#}\right)$ onto $\ell_{\infty}^{u}\left(Æ(X)^{*}\right)=c_{0}\left(Æ(X)^{*}\right)$ for $p=\infty$.

Our next aim is to get a Lipschitz version of the well-known theorem due to Terzioğlu [18, Theorem 1] (see also, [7, Exercise 6, p.15]) and concluded that $\left(\mathcal{C K}_{s t, \infty}^{L}\right)^{\text {inj }}$ coincides with the ideal of Lipschitz compact operators.

Proposition 2.3. Let $T \in \operatorname{Lip}_{0}(X, E)$ between pointed metric space and Banach space. Then the following are equivalent.

i) $T$ is Lipschitz compact.

ii) There exists a norm-null sequence $\left(f_{n}\right)_{n}$ in $X^{\#}$ such that

$$
\left\|\sum_{j=1}^{m} \lambda_{j}\left(T x_{j}-T x_{j}^{\prime}\right)\right\| \leqslant \sup _{n}\left|\sum_{j=1}^{m} \lambda_{j}\left(f_{n}\left(x_{j}\right)-f_{n}\left(x_{j}^{\prime}\right)\right)\right|,
$$

for all $x_{j}, x_{j}^{\prime} \in X$ and $\lambda_{j} \in \mathbb{K}(1 \leq j \leq m)$.

iii) For some closed subspace $M$ of $c_{0}$ with $0 \in M$ there are operators $S \in \operatorname{Lip} p_{0 K}(X, M)$ and $v \in \mathcal{K}(M, E)$ such that $T=v \circ S$.

Proof. i) $\Longleftrightarrow$ ii) We know from [13, Proposition 2.1] that $T$ is Lipschitz compact if and only if $T_{L}$ is compact. By Theorem 1 in [18] there exists a norm null sequence $\left(m_{n}^{*}\right)_{n}$ in $Æ(X)^{*}$, such that

$$
\left\|T_{L}(m)\right\| \leq \sup _{n}\left|m_{n}^{*}(m)\right|, \forall m \in Æ(X)^{*} .
$$

The proof concludes since

$$
\left\|T_{L}(m)\right\|=\left\|T_{L}\left(\sum_{j=1}^{m} \lambda_{j} m_{x_{j} x_{j}^{\prime}}\right)\right\|=\left\|\sum_{j=1}^{m} \lambda_{j}\left(T x_{j}-T x_{j}^{\prime}\right)\right\|,
$$

with $m=\sum_{j=1}^{m} \lambda_{j} m_{x_{j} x_{j}^{\prime}}$ for all $x_{j}, x_{j}^{\prime} \in X$ and $\lambda_{j} \in \mathbb{K}(1 \leq j \leq m)$ and, since $Æ(X)^{*}$ and $X^{\#}$ are isometrically isomorphic, then by Lemma 2.1 (ii), there is a norm-null sequence $\left(f_{n}\right)_{n}$ in $X^{\#}$ such that

$$
m_{n}^{*}\left(m_{x_{j} x_{j}^{\prime}}\right)=\left(f_{n}\right)_{L}\left(m_{x_{j} x_{j}^{\prime}}\right)=f_{n}\left(x_{j}\right)-f_{n}\left(x_{j}^{\prime}\right),
$$

for all $x_{j}, x_{j}^{\prime} \in X, \lambda_{j} \in \mathbb{K},(1 \leq j \leq m)$. 
(i) $\Longleftrightarrow\left(\right.$ iii) Let $T \in \operatorname{Lip}_{0 \mathcal{K}}(X, E)$, then $T=T_{L} \circ \delta_{X}$ with $T_{L} \in \mathcal{K}(Æ(X), E)$. Lemma 3.3 in [10] tells us that (see also, [7]) for some closed subspace $M$ of $c_{0}$ there are compact operators $u: \nexists(X) \longrightarrow M$ and $v: M \longrightarrow E$ such that $T_{L}=v \circ u$. Then $T=v \circ u \circ \delta_{X}$ with $S=u \circ \delta_{X} \in$ $\operatorname{Lip}_{0 \mathcal{K}}(X, M)$ and $v \in \mathcal{K}(M, E)$. Then (iii) follows.

To verify the converse, we let $T=v \circ S$, where $S \in \operatorname{Lip}_{0 \mathcal{K}}(X, M), v \in \mathcal{K}(M, E)$ and $M$ a closed subspace of $c_{0}$. By [13, Proposition 2.1] the operator $S_{L}$ is compact and $\left\|S_{L}\right\|=\operatorname{Lip}(S)$. Then $T=T_{L} \circ \delta_{X}=v \circ S=v \circ S_{L} \circ \delta_{X}$. Since $v \in \mathcal{K}(M, E)$, it follows from uniqueness of linearization that $T_{L}=v \circ S_{L}$ and $T_{L} \in \mathcal{K}(Æ(X), E)$. This proves that $T$ is Lipschitz compact and

$$
\operatorname{Lip}(T)=\left\|T_{L}\right\| \leq\|v\|\left\|S_{L}\right\|=\|v\| \operatorname{Lip}(S) .
$$

Remark 2.1. From Proposition 2.3 and Proposition 2.2, the Lipschitz ideal (Lip 0 K, Lip $(\cdot))$ of Lipschitz compact operators equipped with the Lipschitz norm coincides with $\left(\left(\mathcal{C} \mathcal{K}_{s t, \infty}^{L}\right)^{i n j},\|\cdot\|_{\left(\mathcal{C K}_{s t, \infty}^{L}\right)^{i n j}}\right)$.

\section{Factorable Lipschitz unconditionally quasi $p$-nuclear operators}

If we consider $T \in \operatorname{Lip}_{0}(X, E)$, we obtain a characterization of injective hull of the strongly Lipschitz classical $p$-compact operators as follows.

$$
\begin{aligned}
& T \in\left(\mathcal{C} \mathcal{K}_{s t, p}^{L}\right)^{i n j} \\
& \Longleftrightarrow T_{L} \in \mathcal{N}_{u p}^{Q} \\
& \Longleftrightarrow \text { there exists }\left(f_{n}\right)_{n} \in \ell_{p}^{L, \omega, u}\left(X^{\#}\right) \text { such that } \\
& \left\|\sum_{j=1}^{m} \lambda_{j}\left(T x_{j}-T x_{j}^{\prime}\right)\right\| \leq\left(\sum_{n=1}^{+\infty}\left|\sum_{j=1}^{m} \lambda_{j}\left(f_{n}\left(x_{j}\right)-f_{n}\left(x_{j}^{\prime}\right)\right)\right|^{p}\right)^{\frac{1}{p}} \\
& \text { for all } x_{j}, x_{j}^{\prime} \in X \text { and } \lambda_{j} \in \mathbb{K},(1 \leq j \leq m),
\end{aligned}
$$

where the first equivalence follows Corollary 2.1 and the second one from (2.3). So, among the aims of this section is to contribute to the study of summability of Lipschitz mappings by characterizing those Lipschitz mappings whose its linearizations are unconditionally $p$-nuclear linear operators, namely the classes of factorable Lipschitz unconditionally quasi $p$-nuclear mappings.

Definition 3.1. Let $X$ be pointed metric space, $E$ be a Banach space and $1 \leq p \leq \infty$. A mapping $T \in \operatorname{Lip}_{0}(X, E)$ is called factorable Lipschitz unconditionally quasi p-nuclear operator if there exists a sequence $\left(f_{n}\right)_{n} \in \ell_{p}^{L, \omega^{*}, u}\left(X^{\#}\right)$ such that

$$
\left\|\sum_{j=1}^{m} \lambda_{j}\left(T x_{j}-T x_{j}^{\prime}\right)\right\| \leq\left(\sum_{n=1}^{+\infty}\left|\sum_{j=1}^{m} \lambda_{j}\left(f_{n}\left(x_{j}\right)-f_{n}\left(x_{j}^{\prime}\right)\right)\right|^{p}\right)^{\frac{1}{p}},
$$

for all $x_{j}, x_{j}^{\prime} \in X, \lambda_{j} \in \mathbb{K},(1 \leq j \leq m)$. In such case, we put

$$
v_{f, u p}^{L Q}(T)=\inf \left\{\left\|\left(f_{n}\right)_{n}\right\|_{p}^{L, \omega^{*}}:\left(f_{n}\right)_{n} \text { satisfying (3.2) }\right\} .
$$


We denote by $\mathcal{N}_{f, u p}^{L Q}(X, E)$ the space of all factorable unconditionally quasi p-nuclear Lipschitz mappings between pointed metric spaces $X$ and Banach space $E$.

Comparing this definition with (3.1) yields:

Theorem 3.1. Let $X$ be a pointed metric space, E be a Banach space and $1 \leq p \leq \infty$. A mapping $T \in \operatorname{Lip}_{0}(X, E)$ is factorable Lipschitz unconditionally quasi p-nuclear operator if and only if it belongs to

$$
\left[\left(\mathcal{C K}_{s t, p}^{L}\right)^{i n j},\|\cdot\|_{\left(\mathcal{C K}_{s t, p}^{L}\right)^{i n j}}\right]=\left[\mathcal{N}_{u p}^{Q} \circ \operatorname{Lip}_{0}, v_{u p}^{Q}(\cdot)\right]
$$

In this case $v_{f, u p}^{L Q}(\cdot)=\|\cdot\|_{\left(\mathcal{C K}_{s t, p}^{L}\right)^{i n j}}=v_{u p}^{Q}(\cdot)$.

Proposition 3.1. Let $1 \leq p<\infty$. If $E$ and $F$ are Banach spaces and $T$ is a linear operator, then

$$
\mathcal{C K}_{p}^{i n j}(E, F) \subset\left(\mathcal{C} \mathcal{K}_{s t, p}^{L}\right)^{i n j}(E, F) .
$$

The equality holds when $E$ is separable. Moreover, the norms $\|T\|_{\mathcal{C K}_{p}^{i n j}}$ and $\|T\|_{\left(\mathcal{C K}_{s t, p}^{L}\right)^{\text {inj }}}$ coincides.

Proof. The first implication follows from the inclusion $\ell_{p}^{u}\left(E^{*}\right) \subset \ell_{p}^{L, \omega^{*}, u}\left(E^{\#}\right)$.

Conversely, let $T \in\left(\mathcal{C K}_{s t, p}^{L}\right)^{i n j}(E, F)$ with $E$ separable then $\iota_{F} T \in \mathcal{C} \mathcal{K}_{s t, p}^{L}\left(E, \ell_{\infty}\left(B_{F^{*}}\right)\right)$ with $\|T\|_{\left(\mathcal{C K}_{s t, p}^{L}\right)^{i n j}}=\left\|\iota_{E} T\right\|_{\mathcal{C K}_{s t, p}^{L}}$. By Proposition 2.11 in [5], $\iota_{F} T \in \mathcal{C} \mathcal{K}_{p}\left(E, \ell_{\infty}\left(B_{F^{*}}\right)\right)$ and is a linear operator with $\left\|\iota_{F} T\right\|_{\mathcal{C} \mathcal{K}_{s t, p}^{L}}=\left\|\iota_{F} T\right\|_{\mathcal{C} \mathcal{K}_{p}}$, which implies that $T \in \mathcal{C} \mathcal{K}_{p}^{i n j}(E, F)$ with $\|T\|_{\mathcal{C K}_{p}^{i n j}}=$ $\|T\|_{\left(\mathcal{C} \mathcal{K}_{s t, p}^{L}\right)^{i n j} \text {. }}$

From Remark 2.1, [13, p. 893] and Lemma 3.3 in [10] we get

Proposition 3.2. Four $p=\infty$. If $E$ and $F$ are Banach spaces and $T$ is a linear operator, then

$$
\left(\mathcal{C} \mathcal{K}_{s t, \infty}^{L}\right)^{i n j}(E, F)=\operatorname{Lip}_{0 \mathcal{K}}(E, F)=\mathcal{K}(E, F)=\mathcal{C K}_{\infty}^{i n j}(E, F) .
$$

We finish this section by showing another property of factorable Lipschitz unconditionally quasi $p$-nuclear operators which can be see as an extension of the linear case. Recall that, for $1 \leq p \leq \infty$ a linear operator $T: E \rightarrow F$ is said to be unconditionally $p$-compact (or $u$ - $p$-compact) as in [14] if there exists a sequence $\left(y_{n}\right)_{n} \in \ell_{p}^{u}(F)$ such that

$$
T\left(B_{E}\right) \subset\left\{\sum_{n=1}^{\infty} \alpha_{n} y_{n}:\left(\alpha_{n}\right)_{n} \in B_{\ell_{p^{*}}}\right\} .
$$

The collection of all $u$-p-compact operators from $E$ to $F$ is denoted by $\mathcal{K}_{u p}(E, F)$. A norm $k_{u p}$ is defined on $\mathcal{K}_{u p}(E, F)$ by

$$
k_{u p}(T)=\inf \left\{\left\|\left(y_{n}\right)_{n}\right\|_{p}^{\omega}:\left(y_{n}\right)_{n} \in \ell_{p}^{u}(F) \text { which satisfy (3.3) }\right\} .
$$

The ideal $(\mathcal{K},\|\cdot\|)$ of compact operators equipped with the operator norm coincides with $\left(\mathcal{K}_{u \infty}, k_{u \infty}\right)$ and $\left(\mathcal{K}_{u p}, k_{u p}\right)$ is a Banach operator ideal [14, Theorem 2.1]. In [14, Theorem 2.3] was established the relation between unconditionally $p$-compact operators and unconditionally quasi $p$-nuclear operators. A linear operator $T: E \rightarrow F$ is unconditionally quasi $p$-nuclear if and only if its adjoint $T^{*}: F^{*} \rightarrow E^{*}$ is unconditionally $p$-compact and $v_{u p}^{Q}(T)=k_{u p}\left(T^{*}\right)$. 
For each $T \in \operatorname{Lip}_{0}(X, E)$, the linear operator $T^{t}: E^{*} \longrightarrow X^{\#}$, given by $T^{t} y^{*}=y^{*} \circ T$ for all $y^{*} \in E^{*}$, is called the Lipschitz transpose map of $T$. The norm $\left\|T^{t}\right\|$ is given by $\left\|T^{t}\right\|=\operatorname{Lip}(T)$. If $\left(\mathcal{A},\|\cdot\|_{\mathcal{A}}\right)$ is a Banach ideal, then the Lipschitz dual of $\mathcal{A}$ is defined by Achour et al. [3, Definition 3.8] as follows

$$
\mathcal{A}^{\text {Lip-dual }}(X, E)=\left\{T \in \operatorname{Lip}_{0}(X, E): T^{t} \in \mathcal{A}\left(E^{*}, X^{\#}\right)\right\},
$$

for a pointed metric space $X$ and a Banach space $E$. We define, $\|T\|_{\mathcal{A}^{\text {Lip-dual }}}=\left\|T^{t}\right\|_{\mathcal{A}}$. Then $\left(\mathcal{A}^{\text {Lip-dual }},\|\cdot\|_{\mathcal{A}^{\text {Lip-dual }}}\right)$ becomes a Banach Lipschitz ideal and $\mathcal{A}^{\text {Lip-dual }}(X, E)=\mathcal{A}^{\text {dual }} \operatorname{Lip}_{0}(X, E)$ (see [3, Theorem 3.9]).

By Theorem 2.3 in [14] (see also [10, Proposition 4.7]) and the above criterion, we have the following.

Proposition 3.3. Let $X$ be a pointed metric space, $E$ be a Banach space, and $1 \leq p<\infty$. For an operator $T \in \operatorname{Lip}_{0}(X, E), T^{t}: E^{*} \longrightarrow X^{\#}$ is unconditionally $p$-compact operator if and only if $T \in$ $\left(\mathcal{C} \mathcal{K}_{s t, p}^{L}\right)^{i n j}(X, E)$ and $\|T\|_{\left(\mathcal{C} \mathcal{K}_{s t, p}^{L}\right)^{i n j}}=k_{u p}\left(T^{t}\right)$. In other words, $\left(\mathcal{C K}_{s t, p}^{L}\right)^{i n j}(X, E)=\mathcal{K}_{u p}^{\text {Lip-dual }}(X, E)$ isometrically.

Proof. By Theorem 3.1 and Theorem 2.3 in [14] (see also [10, Proposition 4.7]), we have

$$
\begin{aligned}
\left(\mathcal{C K}_{s t, p}^{L}\right)^{i n j}(X, E) & =\mathcal{N}_{u p}^{Q} \circ \operatorname{Lip}_{0}(X, E) \\
& =\left(\mathcal{K}_{u p}\right)^{\text {dual }} \circ \operatorname{Lip}_{0}(X, E) \\
& =\mathcal{K}_{u p}^{\text {Lip-dual }}(X, E) .
\end{aligned}
$$

The result follows.

\section{The Lipschitz injective hull of Lipschitz classical $p$-compact operators}

Now, we center our study in the case when the Lipschitz operators are consider between pointed metric spaces. In this section, we consider an extended version of the unconditionally quasi $p$-nuclear operators, we called it the Lipschitz unconditionally quasi $p$-nuclear. It is well known that in the linear case, the ideal of linear unconditionally quasi $p$-nuclear operators coincide with injective hull of the Banach operator ideal of classical $p$-compact operators [10]. Then we show that this result can be extended to the Lipschitz case.

The Lipschitz $(p, r, s)$-nuclear operators were introduced by Belacel and Chen in [5] and can be extended in a verbatim way to Lipschitz maps between pointed metric spaces. For pointed metric spaces $X$ and $Y$, suppose that $1+\frac{1}{p} \geq \frac{1}{r}+\frac{1}{s}$, a Lipschitz mapping $T \in \operatorname{Lip}_{0}(X, Y)$ is called Lipschitz $(p, r, s)$-nuclear, if there exist two Lipschitz mappings $A \in \operatorname{Lip}_{0}\left(\ell_{r}, Y\right)$ and $B \in \operatorname{Lip}_{0}\left(X, \ell_{S^{*}}\right)$ and a sequence $\lambda \in \ell_{p}$ such that the following diagram commute

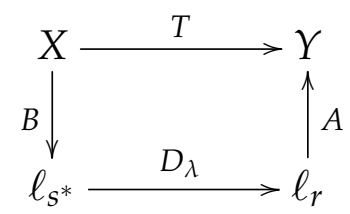


where $D_{\lambda}: \ell_{s^{*}} \longrightarrow \ell_{r}$ is the diagonal operator induced by the sequence $\lambda \in \ell_{p}$. The set of all Lipschitz $(p, r, s)$-nuclear operators from $X$ to $Y$ is denoted by $\mathcal{N}_{(p, r, s)}^{L}(X, Y)$. To the class $\mathcal{N}_{(p, r, s)}^{L}(X, Y)$ we will assign the function $\|\cdot\|_{\mathcal{N}_{(p, r, s)}^{L}}$ defined, for $T \in \mathcal{N}_{(p, r, s)}(X, Y)$, as the infimum of $\operatorname{Lip}(A)\|\lambda\|_{p} \operatorname{Lip}(B)$ over all factorizations as in (4.1).

Let $1 \leq p \leq \infty$ and $T \in \operatorname{Lip}_{0}(X, Y)$. We say that $T$ is Lipschitz classical $p$-compact if it belongs to

$$
\left[\mathcal{C K}_{p}^{L},\|\cdot\|_{\mathcal{C} \mathcal{K}_{p}^{L}}\right]:=\left[\mathcal{N}_{\left(\infty, p, p^{*}\right)}^{L},\|\cdot\|_{\mathcal{N}_{\left(\infty, p, p^{*}\right)}^{L}}\right] .
$$

Following [1] an ideal of Lipschitz maps $\mathcal{I}_{\text {Lip }}$ between pointed metric spaces we mean an assignment for each pair of pointed metric spaces $X$ and $Y$ of a family of mappings $\mathcal{I}_{\text {Lip }}(X, Y) \subset \operatorname{Lip}_{0}(X, Y)$, together with a real-valued function $\|\cdot\|_{\mathcal{I}_{\text {Lip }}}$ which satisfies:

i) If $Y=E$ is a Banach space, then for any $f \in X^{\#}$ and $e \in E$, the map $f e: x \mapsto f(x) e \in$ $\mathcal{I}_{\text {Lip }}(X, E)$.

ii) For every $T \in \mathcal{I}_{\text {Lip }}(X, Y)$, $\operatorname{Lip}(T) \leq\|T\|_{\mathcal{I}_{\text {Lip }}}$.

iii) The ideal property: If $R \in \operatorname{Lip}_{0}\left(X_{1}, X\right), T \in \mathcal{I}_{L i p}(X, Y)$ and $S \in \operatorname{Lip}_{0}\left(Y, Y_{1}\right)$, then $S T R \in$ $\mathcal{I}_{\text {Lip }}\left(X_{1}, Y_{1}\right)$ and $\|S T R\|_{\mathcal{I}_{\text {Lip }}} \leq \operatorname{Lip}(S)\|T\|_{\mathcal{I}_{\text {Lip }}} \operatorname{Lip}(R)$.

Proposition 4.1. For $1 \leq p \leq \infty, \mathcal{C} \mathcal{K}_{p}^{L}$ is a Lipschitz operator ideal.

Proof. i) As $\mathcal{C} \mathcal{K}_{s t, p}^{L}(X, E)$ is a Banach Lipschitz operator ideal and

$$
\mathcal{C K}_{s t, p}^{L}(X, E) \subset \mathcal{C} \mathcal{K}_{p}^{L}(X, E),
$$

then $f(x) e \in \mathcal{C K}_{p}^{L}(X, E)$ for any $f \in X^{\#}$ and $e \in E$.

ii) Let $T \in \mathcal{C K}_{p}^{L}(X, Y)$ and $\varepsilon>0$. So, (4.1) implies that

$$
\begin{aligned}
\operatorname{Lip}(T) & =\operatorname{Lip}\left(A D_{\lambda} B\right) \\
\leq & \operatorname{Lip}(A)\|\lambda\|_{\infty} \operatorname{Lip}(B) \\
\leq & (1+\varepsilon)\|T\|_{\mathcal{C K}_{p}^{L} .}
\end{aligned}
$$

Letting $\varepsilon \longrightarrow 0$, we get $\operatorname{Lip}(T) \leq\|T\|_{\mathcal{C K}_{p}^{L}}$.

iii) Let $R \in \operatorname{Lip}_{0}\left(X_{1}, X\right), T \in \mathcal{C} \mathcal{K}_{p}^{L}(X, Y)$ and $S \in \operatorname{Lip}_{0}\left(Y, Y_{1}\right)$. For $\varepsilon>0$, take $T=A D_{\lambda} B$ with $B \in \operatorname{Lip}_{0}\left(X, \ell_{p}\right), A \in \operatorname{Lip}_{0}\left(\ell_{p}, Y\right)$, and $\operatorname{Lip}(A)\|\lambda\|_{\infty} \operatorname{Lip}(B) \leq(1+\varepsilon)\|T\|_{\mathcal{C} \mathcal{K}_{p}^{L}}$. Thus, we have $S T R=S A D_{\lambda} B R=A_{1} D_{\lambda} B_{1}$ where $B_{1}=B R \in \operatorname{Lip}_{0}\left(X_{1}, \ell_{p}\right), A_{1}=S A \in \operatorname{Lip}_{0}\left(\ell_{p}, Y_{1}\right)$ and

$$
\begin{aligned}
\|S T R\|_{\mathcal{C K}}^{L} & =\operatorname{Lip}\left(A_{1}\right)\|\lambda\|_{\infty} \operatorname{Lip}\left(B_{1}\right) \\
& \leq \operatorname{Lip}(A)\|\lambda\|_{\infty} \operatorname{Lip}(B) \\
& \leq \operatorname{Lip}(S)(1+\varepsilon)\|T\|_{\mathcal{C K}_{p}^{L}} \operatorname{Lip}(R) .
\end{aligned}
$$

Letting $\varepsilon \longrightarrow 0$, we get $\|S T R\|_{\mathcal{C K}_{p}^{L}} \leq \operatorname{Lip}(S)\|T\|_{\mathcal{C K}_{p}^{L}} \operatorname{Lip}(R)$. 
From Proposition 2.2 in [5] and [16, Definition 18.3.1 ], we have.

Proposition 4.2. Let $1 \leq p<\infty$. Let $T$ be a bounded linear operator from a separable Banach space $E$ into a dual space $F$. Then

$$
\mathcal{C K}_{p}^{L}(E, F)=\mathcal{N}_{\left(\infty, p, p^{*}\right)}(E, F):=C \mathcal{K}_{p}(E, F) .
$$

Moreover, the norms $\|T\|_{\mathcal{C K}_{p}^{L}}$ and $\|T\|_{\mathcal{C} \mathcal{K}_{p}}$ coincides.

Now we give the first characterization of Lipschitz classical $p$-compact operators which extend the results of the linear case.

Theorem 4.1. Let $X$ and $Y$ be pointed metric spaces and $1 \leq p \leq \infty$. For a mapping $T \in \operatorname{Lip}_{0}(X, Y)$, the following are equivalent.

(1) $T \in \mathcal{C K}_{p}^{L}(X, Y)$.

(2) $T$ has a factorization $T=R S$, where $S \in \operatorname{Lip} p_{0 K}\left(X, \ell_{p}\right)$ and $R \in \operatorname{Lip}_{0}\left(\ell_{p}, Y\right)$.

Moreover, $\|T\|_{\mathcal{C K}_{p}^{L}}=\inf \{\operatorname{Lip}(R) \operatorname{Lip}(S): T=R \circ S\}$ where the infimum is taken over all factorizations as above.

Proof. (1) $\Longrightarrow(2)$ Let $\varepsilon>0$ and take $T \in \mathcal{C} \mathcal{K}_{p}^{L}(X, Y)$, then there exist two Lipschitz operators $B \in \operatorname{Lip}_{0}\left(X, \ell_{p}\right), A \in \operatorname{Lip}_{0}\left(\ell_{p}, Y\right)$ and a diagonal operator $D_{\lambda} \in \mathcal{L}\left(\ell_{p}, \ell_{p}\right)$ such that $T=A D_{\lambda} B$, where $\lambda \in \mathcal{c}_{0}$ and $\operatorname{Lip}(A)\|\lambda\|_{\infty} \operatorname{Lip}(B) \leq(1+\varepsilon)\|T\|_{\mathcal{C K}_{p}^{L}}$. If we consider the operator $S=D_{\lambda} B$, since $D_{\lambda}$ is a compact linear operator, $S$ is Lipschitz compact. Let $R=A$ and $S=D_{\lambda} B$. Thus, $T=A D_{\lambda} B=R S$ and

$$
\begin{aligned}
\operatorname{Lip}(R) \operatorname{Lip}(S) & \leq \operatorname{Lip}(A)\|\lambda\|_{\infty} \operatorname{Lip}(B) \\
& \leq(1+\varepsilon)\|T\|_{\mathcal{C K}_{p}^{L}} .
\end{aligned}
$$

Letting $\varepsilon \longrightarrow 0$, we get inf $\operatorname{Lip}(R) \operatorname{Lip}(S) \leq\|T\|_{\mathcal{C K}_{p}^{L}}$.

$(2) \Longrightarrow(1)$ Suppose that $T$ has a factorization $T=R S$, where $S \in \operatorname{Lip} p_{0 \mathcal{K}}\left(X, \ell_{p}\right)$ and $R \in$ $\operatorname{Lip}_{0}\left(\ell_{p}, Y\right)$, then $S_{L}: Æ(X) \longrightarrow \ell_{p}$ is compact and $\left\|S_{L}\right\|=\operatorname{Lip}(S)$. By [11, Proposition 2.2 (a)], we get $\lambda=\left(\lambda_{n}\right)_{n} \in c_{0},\|\lambda\|=1$ and an operator $\hat{S_{L}} \in \mathcal{K}\left(Æ(X), \ell_{p}\right)$ such that $S_{L}=D_{\lambda} \hat{S_{L}}$ and $\left\|\hat{S_{L}}\right\| \leq(1+\varepsilon)\left\|S_{L}\right\|$ for $\varepsilon>0$. Hence $T=R D_{\lambda} \hat{S_{L}} \delta_{X}=A D_{\lambda} B$ with $A=R \in \operatorname{Lip}_{0}\left(\ell_{p}, Y\right)$ and $B=\hat{S_{L}} \delta_{X} \in \operatorname{Lip}_{0}\left(X, \ell_{p}\right)$. Then $T \in \mathcal{C} \mathcal{K}_{p}^{L}$ and $\|T\|_{\mathcal{C} \mathcal{K}_{p}^{L}} \leq(1+\varepsilon) \operatorname{Lip}(R) \operatorname{Lip}(S)$.

Letting $\varepsilon \longrightarrow 0$, we get $\|T\|_{\mathcal{C K}_{p}^{L}} \leq \inf \operatorname{Lip}(R) \operatorname{Lip}(S)$.

The notion of unconditionally quasi p-nuclear operators was introduced by Kim [14, p. 136]. Now, we extend this notion to the Lipschitz case.

Definition 4.1. Let $X$ and $Y$ be pointed metric spaces and $1 \leq p \leq \infty$. A mapping $T \in \operatorname{Lip}_{0}(X, Y)$ is called Lipschitz unconditionally quasi -nuclear operator if there exists a sequence $\left(f_{n}\right)_{n} \in \ell_{p}^{L, \omega^{*}, u}\left(X^{\#}\right)$ such that

$$
d\left(T x, T x^{\prime}\right) \leq\left\|\left(f_{n}(x)-f_{n}\left(x^{\prime}\right)\right)_{n}\right\|_{p}
$$


for all $x, x^{\prime} \in X$. In such case, we put

$$
v_{u p}^{L Q}(T)=\inf \left\{\left\|\left(f_{n}\right)_{n}\right\|_{p}^{L, \omega^{*}}:\left(f_{n}\right)_{n} \text { satisfying }(4.2)\right\} .
$$

The class of all Lipschitz unconditionally quasi p-nuclear mappings from $X$ to $Y$ is denoted by $\mathcal{N}_{u p}^{L Q}(X, Y)$. It is easily seen that $\operatorname{Lip}(T) \leq v_{\text {up }}^{L Q}(T)$ for all $T \in \mathcal{N}_{\text {up }}^{L Q}(X, Y)$.

Proposition 4.3. Let $1 \leq p \leq \infty, X$ be a pointed metric space, $E$ be a Banach space, and $T \in$ $\operatorname{Lip}_{0}(X, E)$. Then

$$
\left(\mathcal{C K}_{s t, p}^{L}\right)^{i n j}(X, E) \subset \mathcal{N}_{u p}^{L Q}(X, E)
$$

and $v_{u p}^{L Q}(T) \leq\|T\|_{\left(\mathcal{C} \mathcal{K}_{s t, p}^{L}\right)^{i n j}}$ for all $T \in\left(\mathcal{C} \mathcal{K}_{s t, p}^{L}\right)^{i n j}(X, E)$.

Proof. If $T \in\left(\mathcal{C K}_{s t, p}^{L}\right)^{i n j}(X, E)$, by Corollary $2.1 T_{L} \in \mathcal{C} \mathcal{K}_{p}^{i n j}(Æ, E)$. Also, by Theorem 4.4 in [10] there exists a sequence $\left(m_{n}^{*}\right)_{n} \in \ell_{p}^{u}\left(Æ(X)^{*}\right)$ such that $\left\|T_{L}(m)\right\| \leq\left\|\left(m_{n}^{*}(m)\right)_{n}\right\|_{p}$ for all $m \in Æ(X)$. The proof concludes since $\left\|T x-T x^{\prime}\right\|=\left\|T_{L}\left(m_{x x^{\prime}}\right)\right\|$, and since $X^{\#}$ is isometrically isomorphic to $Æ(X)^{*}$, for each $m_{n}^{*} \in Æ(X)^{*}$ there is $f_{n} \in X^{\#}$ with $\operatorname{Lip}\left(f_{n}\right)=\left\|m_{n}^{*}\right\|$ such that

$$
m_{n}^{*}\left(m_{x x^{\prime}}\right)=f_{n}(x)-f_{n}\left(x^{\prime}\right),
$$

for all $x, x^{\prime} \in X$ and all $n \in \mathbb{N}$.

Question. Does $T \in \operatorname{Lip}_{0}(X, E)$ is Lipschitz unconditionally quasi $p$-nuclear imply $T \in$ $\left(\mathcal{C K}_{\text {st, }}^{L}\right)^{\operatorname{inj}}(X, E)$.

In [1], the authors introduced the Lipschitz injective hull of a Lipschitz operator ideal. Let $\mathcal{I}_{\text {Lip }}$ be a Lipschitz operator ideal between pointed metric spaces. For pointed metric spaces $X$ and $Y$, a Lipschitz operator $T \in \operatorname{Lip}_{0}(X, Y)$ belongs to the Lipschitz injective hull of $\mathcal{I}_{\text {Lip }}$ if there exists a pointed metric space $Z$ and a Lipschitz operator $S \in \mathcal{I}_{\text {Lip }}(X, Z)$ such that $d\left(T x, T x^{\prime}\right) \leq d\left(S x, S x^{\prime}\right)$ for all $x, x^{\prime} \in X$. The class of all operators from $X$ to $Y$ which belongs to the Lipschitz injective hull of $\mathcal{I}_{\text {Lip }}$ will be denoted by $\mathcal{I}_{\text {Lip }}^{\text {Linj }}(X, Y)$. Recall that for every pointed metric space $X$ is isometric to a $\ell_{\infty}(\Gamma)$ for some set $\Gamma$ (see for instance [6, Lemma 1.1]). We my consider an isometry which maps 0 to 0 , denoted by $\iota_{Y}^{L_{0}}: Y \longrightarrow \ell_{\infty}(\Gamma)$. Then, following [1, Proposition 1.2], for a Lipschitz operator ideal between pointed metric spaces $\mathcal{I}_{\text {Lip }}$, a Lipschitz operator $T: X \rightarrow Y$ belongs to $\mathcal{I}_{\text {Lip }}^{\text {Linj }}(X, Y)$ if and only if $\iota_{Y}^{L_{0}} T$ belongs to $\mathcal{I}_{\text {Lip }}\left(X, \ell_{\infty}(\Gamma)\right)$ with $\|T\|_{\mathcal{I}_{\text {Lip }}^{\text {Linj }}}=\left\|\iota_{Y}^{L_{0}} T\right\|_{\mathcal{I}_{\text {Lip }}}$. If $\mathcal{I}_{\text {Lip }}$ is a Lipschitz operator ideal, then $\mathcal{I}_{\text {Lip }}^{\text {Linj }}$ is also a Lipschitz operator ideal.

We now turn to a discussion of the Lipschitz injective hull of the Lipschitz operator ideal $\mathcal{C} \mathcal{K}_{p}^{L}$.

Theorem 4.2. Let $X$ and $Y$ be pointed metric spaces and $1 \leq p \leq \infty$. For a mapping $T \in \operatorname{Lip}_{0}(X, Y)$, the following are equivalent.

(a) $T \in\left(\mathcal{C K}_{p}^{L}\right)^{\operatorname{Linj}}(X, Y)$. 
(b) $T \in \mathcal{N}_{u p}^{L Q}(X, Y)$.

$$
\text { Moreover, } v_{\text {up }}^{L Q}(T)=\|T\|_{\left(\mathcal{C} \mathcal{K}_{p}^{L}\right)^{L i n j} \text {. }}
$$

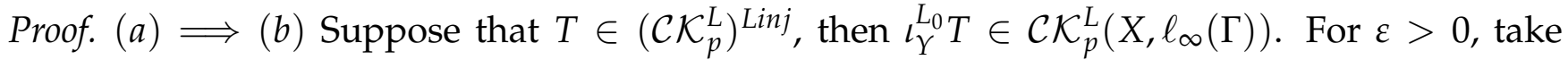
$\iota_{Y}^{L_{0}} T=A D_{\lambda} B$ with $B \in \operatorname{Lip}_{0}\left(X, \ell_{p}\right), A \in \operatorname{Lip}{ }_{0}\left(\ell_{p}, \ell_{\infty}(\Gamma)\right), \operatorname{Lip}(A)=\operatorname{Lip}(B)=1$ and $\|\lambda\|_{\infty} \leq$

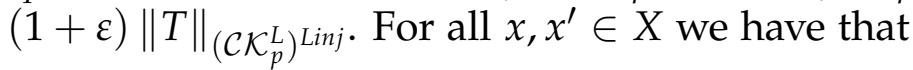

$$
\begin{aligned}
d\left(T x, T x^{\prime}\right) & =d\left(\iota_{Y}^{L_{0}} T x, \iota_{Y}^{L_{0}} T x^{\prime}\right)=d\left(A D_{\lambda} B x, A D_{\lambda} B x^{\prime}\right) \\
& \leq \operatorname{Lip}(R)\left\|D_{\lambda}\left(B x-B x^{\prime}\right)\right\|_{p} \\
& =\left(\sum_{n=1}^{+\infty}\left|\lambda_{n} \zeta_{n}\left(B x-B x^{\prime}\right)\right|^{p}\right)^{\frac{1}{p}} \\
& =\left(\sum_{n=1}^{+\infty}\left|\lambda_{n} \zeta_{n} B x-\lambda_{n} \zeta_{n} B x^{\prime}\right|^{p}\right)^{\frac{1}{p}} .
\end{aligned}
$$

Here, $\left(\zeta_{n}\right)_{n}$ stands for the sequence of coordinate functional on $\ell_{p}$. Denoting by $f_{n}=\lambda_{n} \zeta_{n} B$, it follows that $\left(f_{n}\right)_{n} \in \ell_{p}^{L, \omega^{*}, u}\left(X^{\#}\right)$ by Lemma 2.4 in [5] and $\left\|\left(f_{n}\right)_{n}\right\|_{p}^{L, \omega^{*}}=\left\|\left(\lambda_{n}\right)_{n}\right\|_{\infty}$, which implies that $T$ is Lipschitz unconditionally quasi $p$-nuclear operator and $v_{u p}^{L Q}(T) \leq\left\|\left(\lambda_{n}\right)_{n}\right\|_{\infty} \leq$ $(1+\varepsilon)\|T\|_{\left(\mathcal{C K}_{p}^{L}\right) \text { Linj }}$.

$(b) \Longrightarrow(a)$ Assume that $T$ is a Lipschitz unconditionally quasi $p$-nuclear and $\varepsilon>0$ be given. Then there exists a sequence $\left(f_{n}\right)_{n} \in \ell_{p}^{L, \omega^{*}, u}\left(X^{\#}\right)$ such that $d\left(T x, T x^{\prime}\right) \leq\left\|\left(f_{n}(x)-f_{n}\left(x^{\prime}\right)\right)_{n}\right\|_{p}$ for all $x, x^{\prime} \in X$ and $\left\|\left(f_{n}\right)_{n}\right\|_{p}^{L, \omega^{*}} \leq(1+\varepsilon) v_{u p}^{L Q}(T)$. Define the mapping $S: X \longrightarrow \ell_{p}$ by $S x=\left(f_{n}(x)\right)_{n}$. It follows from Lemma 3.5 in [5] that $S$ is Lipschitz compact and $\operatorname{Lip}(S)=$ $\left\|\left(f_{n}\right)_{n}\right\|_{p}^{L, \omega^{*}}$. Consider the set $Z_{0}=S(X)$ of $\ell_{p}$ containing 0 and the map $R_{0}: Z_{0} \longrightarrow Y$ by $R_{0}(S x) \longmapsto T x, x \in X$. Clearly, $R_{0}$ is well-defined Lipschitz mapping with $R_{0}(0)=0$ and $\operatorname{Lip}\left(R_{0}\right) \leq 1$. Since $\ell_{\infty}(\Gamma)$ is injective, the operator $L_{Y}^{L_{0}} R_{0}$ extends to a Lipschitz operator $R: \ell_{p} \longrightarrow \ell_{\infty}(\Gamma)$ such that $\operatorname{Lip}\left(\iota_{Y}^{L_{0}} R_{0}\right)=\operatorname{Lip}(R)$ [6, Proposition 1.2] and $\iota_{Y}^{L_{0}} T=R \circ S$. By Theorem 4.1 we have $\iota_{Y}^{L_{0}} T \in \mathcal{C} \mathcal{K}_{p}^{L}\left(X, \ell_{\infty}(\Gamma)\right)$ and $\left\|\iota_{Y}^{L_{0}} T\right\|_{\mathcal{C K}_{p}^{L}} \leq(1+\varepsilon) \operatorname{Lip}(R) \operatorname{Lip}(S)$ for $\varepsilon>0$. So, $T \in\left(\mathcal{C K}_{p}^{L}\right)^{\operatorname{Linj}}(X, Y)$ and $\|T\|_{\left(\mathcal{C K}_{p}^{L}\right)^{L i n j}} \leq(1+\varepsilon)\left\|\left(f_{n}\right)_{n}\right\|_{p}^{L, \omega^{*}}$.

Letting $\varepsilon \longrightarrow 0$, we get $\|T\|_{\left(\mathcal{C} \mathcal{K}_{p}^{L}\right)^{L i n j}} \leq\left\|\left(f_{n}\right)_{n}\right\|_{p}^{L, \omega^{*}}$. The proof conclude.

From Proposition 1.2 in [1], we get.

Corollary 4.1. Let $X$ and $Y$ be pointed metric spaces, where $Y$ is injective. Then $\mathcal{C K}_{p}^{L}(X, Y)=$ $\left(\mathcal{C} \mathcal{K}_{p}^{L}\right)^{\operatorname{Linj}}(X, Y)$ with $\|T\|_{\mathcal{C K}_{p}^{L}}=\|T\|_{\left(\mathcal{C} \mathcal{K}_{p}^{L}\right)^{L i n j} \text {. }}$ 
In the following result, we can show that the Lipschitz unconditionally quasi $p$-nuclear norm is equal to the unconditionally quasi $p$-nuclear norm for a linear operator whose domain is separable.

Proposition 4.4. Let $1 \leq p<\infty, E$ and $F$ be Banach spaces and $T \in \mathcal{L}(E, F)$. Then

$$
\mathcal{C} \mathcal{K}_{p}^{i n j}(E, F)=\mathcal{N}_{u p}^{Q}(E, F) \subset \mathcal{N}_{u p}^{L Q}(E, F)=\left(\mathcal{C K}_{p}^{L}\right)^{\operatorname{Linj}}(E, F) .
$$

The equality holds when $E$ is separable. Moreover, the norms $v_{u p}^{L Q}$ and $v_{u p}^{Q}$ coincides.

Proof. The first implication follows from the inclusion $\ell_{p}^{u}\left(E^{*}\right) \subset \ell_{p}^{u}\left(E^{\#}\right)=\ell_{p}^{L, \omega^{*}, u}\left(E^{\#}\right)$. For the converse, suppose that $E$ is separable and $T$ is Lipschitz unconditionally quasi $p$-nuclear. We consider the linear isometry $\iota_{F}: F \longrightarrow \ell_{\infty}\left(B_{F^{*}}\right)$, then by Theorem 4.2, the mapping $\iota_{F} T: E \longrightarrow$ $\ell_{\infty}\left(B_{F^{*}}\right)$ is a linear operator which is Lipschitz classical $p$-compact operator with $v_{u p}^{L Q}(T)=$ $\left\|\iota_{F} T\right\|_{\mathcal{C K}_{p}^{L}}$. Since $E$ is separable and $\ell_{\infty}\left(B_{F^{*}}\right)$ is a dual space, then by Proposition $4.2, \iota_{F} T$ is a classical $p$-compact with $\left\|\iota_{F} T\right\|_{\mathcal{C} \mathcal{K}_{p}}=\left\|\iota_{F} T\right\|_{\mathcal{C K}_{p}^{L}}$, which implies that $T \in \mathcal{C} \mathcal{K}_{p}(E, F)^{i n j}=$ $\mathcal{N}_{u p}^{Q}(E, F)$ by [10, Theorem 4.4] and $v_{u p}^{Q}(T)=v_{u p}^{L Q}(T)$.

The next result establishes the relation of unconditionally quasi $p$-nuclear mapping $T: X \rightarrow$ $Y$ and its Lipschitz adjoint $T^{\#}: Y^{\#} \longrightarrow X^{\#}$. That is, $T^{\#} \in \mathcal{L}\left(Y^{\#}, X^{\#}\right)$ is the linear operator given by $T^{\#}(g)=g \circ T$ for all $g \in Y^{\#}$.

Proposition 4.5. Let $X$ and $Y$ be pointed metric spaces and $1 \leq p<\infty$. For an operator $T \in$ $\operatorname{Lip}_{0}(X, Y)$, if $T^{\#}: Y^{\#} \longrightarrow X^{\#}$ is unconditionally p-compact linear operator, then $T \in \mathcal{N}_{u p}^{L Q}(X, Y)$ and $v_{\text {up }}^{L Q}(T) \leq k_{\text {up }}\left(T^{\#}\right)$.

Proof. Notice first that for all $x, x^{\prime} \in X$ we have

$$
d\left(T x, T x^{\prime}\right)=\sup _{g \in B_{\Upsilon^{\#}}}\left|g(T x)-g\left(T x^{\prime}\right)\right|=\sup _{g \in B_{\Upsilon^{\#}}}\left|\left(T^{\#} g\right)(x)-\left(T^{\#} g\right)\left(x^{\prime}\right)\right| .
$$

Now, suppose that $T^{\#}$ is unconditionally $p$-compact and take $\varepsilon>0$. Choose a sequence $\left(f_{n}\right)_{n} \in \ell_{p}^{u}\left(X^{\#}\right)$ such that $T^{\#}\left(B_{Y^{\#}}\right) \subset\left\{\sum_{n=1}^{\infty} \alpha_{n} f_{n}:\left(\alpha_{n}\right)_{n} \in B_{\ell_{p^{*}}}\right\}$ and $\left\|\left(f_{n}\right)_{n}\right\|_{p}^{L, \omega^{*}} \leq \varepsilon+k_{u p}\left(T^{\#}\right)$. For a fixed $g \in B_{Y^{\#}}$ there exists a sequence $\left(\alpha_{n}\right)_{n} \in B_{\ell_{p^{*}}}$ such that $T^{\#} g=\sum_{n=1}^{\infty} \alpha_{n} f_{n}$. An application of Hölder's inequality reveals that

$$
\begin{aligned}
\left|\left(T^{\#} g\right)(x)-\left(T^{\#} g\right)\left(x^{\prime}\right)\right| & =\left|\sum_{n=1}^{\infty} \alpha_{n} f_{n}(x)-\sum_{i=1}^{\infty} \alpha_{n} f_{n}\left(x^{\prime}\right)\right| \\
& =\mid \sum_{n=1}^{\infty} \alpha_{n}\left(f_{n}(x)-f_{n}\left(x^{\prime}\right) \mid\right. \\
& \leq\left(\sum_{n=1}^{\infty}\left|f_{n}(x)-f_{n}\left(x^{\prime}\right)\right|^{p}\right)^{\frac{1}{p}} .
\end{aligned}
$$


By taking the supremum over all $g \in B_{Y^{\#}}$ we obtain that $T \in \mathcal{N}_{u p}^{L Q}(X, Y)$ with $v_{u p}^{Q L}(T) \leq$ $\left\|\left(f_{n}\right)_{n}\right\|_{p}^{L, \omega^{*}} \leq \varepsilon+k_{u p}\left(T^{\#}\right)$ and the proof follows.

\section{Acknowledgments}

We would like to thank the referee for his/her careful reading of the mansuscript and for his/her suggestions. Also, we acknowledge with thanks the support of the General Direction of Scientific Research and Technological Development (DGRSDT), Algeria.

\section{References}

[1] D. Achour, E. Dahia and P. Turco, The Lipschitz injective hull of Lipschitz operator ideals and applications, Banach J. Math. Anal, 14 (2020), 1241-1257.

[2] D. Achour, E. Dahia and P. Turco, Lipschitz p-compact mapping, Monatshefte für Mathematik, 189 (2019), 595-609.

[3] D. Achour, P. Rueda, E. A. Sanchez-Perez and R. Yahi, Lipschitz operator ideals and the approximation property, J. Math. Anal. Appl., 436, 1 (2016), 217-236.

[4] R. F. Arens and J. Eells Jr, On embedding uniform and topological spaces, Pacific J. Math., 6 (1956), 397-403.

[5] A. Belacel and D. Chen, Lipschitz $(p, r, s)$-integral operators and Lipschitz $(p, r, s)$-nuclear operators, J. Math. Anal. Appl., 461, 2 (2018), 1115-1137.

[6] Y. Benyamini and J. Lindenstrauss, Geometric nonlinear functional analysis, vol. 1. Amer. Math. Soc. Colloq. Publ., vol. 48, Amer. Math. Soc. Providence, RI, (2000).

[7] J. Diestel, Sequences and Series in Banach Spaces, Grad. Texts in Math. 92, Springer, New York (1984).

[8] J. D. Farmer and W.B. Johnson, Lipschitz p-summing operators, Proc. Amer. Math. Soc., 137, 9 (2009), 29892995.

[9] J. H. Fourie, Quasi- and pseudo $\Lambda$-compact operators and locally convex spaces, Tamkang J. Math., 14 (1983), 171-181.

[10] J.H. Fourie, Injective and surjective hulls of classical $p$-compact operators with application to unconditionally p-compact operators, Studia Math., 240 (2018), 147-159.

[11] J.H. Fourie and J. Swart, Banach ideal of $p$-compact operators, Manuscripta Math., 26 (1979), 349-362.

[12] G. Godefroy, A survey on Lipschitz-free Banach spaces, Commentationes Mathematicae, 55, 2 (2015), 89-118.

[13] A. Jiménez-Vargas, J.M. Sepulcre and Moisés Villegas-Vallecillos, Lipschitz compact operators, J. Math. Anal. Appl., 415, 2 (2014), 889-901.

[14] J.M. Kim, Unconditionally $p$-null sequences and unconditionally p-compact operators, Studia Math., 224 (2014), 133-142.

[15] E. Oja, A remark on the approximation of $p$-compact operators by finite-rank operators, J. Math. Anal. Appl., 387, 2 (2012), 949-952.

[16] A. Pietsch, Operator Ideals, Deutsch. Verlag Wiss, Berlin (1978), North-Holland, Amsterdam-London-New York-Tokyo (1980).

[17] D. P. Sinha and A. K. Karn, Compact operators whose adjoints factor through subspaces of $\ell_{p}$, Studia Math., 150 (2002), 17-33.

[18] T. Terzioğlu, A characterization of compact linear mappings, Arch. Math., (Basel) 22 (1971), 76-78.

[19] N. Weaver, Lipschitz Algebras, World Scientific Publishing Co, Singapore (1999). 